\title{
Effect of condensing environments on characteristics of piezoresistive ceramic pressure sensors
}

\author{
Marina Santo Zarnik*, Franc Novak \\ Jozef Stefan Institute, Jamova cesta 39, 1000 Ljubljana, Slovenia
}

\section{A R T I C L E I N F O}

\section{Article history:}

Received 5 June 2017

Received in revised form 6 September 2017

Accepted 13 October 2017

Available online 16 October 2017

\section{Keywords:}

LTCC

Humidity

Harsh environment

Maintenance

\begin{abstract}
A B S T R A C T
A humid atmosphere with water drops is among the most problematic environments for pressure sensors. In general the sensors are protected against harmful effects of media with conformal coatings which, however, in some way affect the characteristics of the sensor. LTCC technology enables manufacturing of the sensing structures that may operate in harsh environments without additional protection. Independently of the implemented protection we should be aware of the changes in the sensor response to the occasional contact with water drops, which result from the changed thermal conditions in the sensing structure. Our experiments demonstrated the influence of the protective coating on the response of the sensor exposed to a humid environment or immersed in the water as well as the effects of the changes in the response due to the water drops applied on the sensor without protection. Appropriate measures taken on the basis of forecasting sensor operation in condensing environments are a part of proactive system maintenance.
\end{abstract}

(C) 2017 Elsevier B.V. All rights reserved.

\section{Introduction}

The characteristics of ceramic pressure sensors mainly depend on the geometry of the sensing structure, the material's properties, and the number of design/technological parameters. Besides, the effect of the surrounding atmosphere/fluid may also change the sensor's offset and its response to the pressure loads [1]. This aspect is important and should also be considered when assessing sensor operation conditions in practice. From the use cases collected within our work in the field of system maintenance, it is apparent that there are cases where it is necessary to provide direct contact with the harsh environments, e.g. pressure measurements of soiled and moist air, condensing environment, the presence of drops or even small quantities of liquids. In these cases, specific solutions with the sensors closed in appropriate housings preventing direct contact of some parts with the environment are employed. They are designed for the specific operating conditions and usually relatively expensive. Apart from them, numerous other sensors are also employed, where it is assumed that they will not be exposed to a harsh environment. Yet, one cannot exclude such situation and for this case it is helpful to predict their response.

\footnotetext{
* Corresponding author.

E-mail addresses: marina.santo@ijs.si (M.S. Zarnik),Franc.novak@ijs.si (F. Novak).
}

In this paper we present some specific examples of ceramic sensors submitted to the above mentioned environmental conditions and point to the effects that can be problematic in normal sensor operation. We focus on low temperature co-fired ceramic (LTCC) pressure sensors because we have practical experience in their design. LTCC is a well-established technology for ceramic components/systems based on processing of green glass ceramic tape. Because of its desirable physical, electrical, and chemical properties coupled with its capability for 3D structuring and scalable manufacturing of different components, LTCC has proven to be an enabling material for micro/meso-system technology [2,3]. Among the most representative LTCC-based microsystems are different sensors for mechanical quantities [4-7]. Low thermal conduction enables implementation of functional structures featuring local high temperature regions such as fluid structure with heaters in a flow sensor [5], DSC calorimeter [8] and others. Previously we investigated the influence of the humidity/water on the sensor response from the long-term stability point of view $[9,10]$ and the impact of the housing on the characteristics of the ceramic pressure sensors $[11,12]$. Now we present our work related to the specific effects of humid/condensing environments. The novelty are dynamic measurements of the sensors immersed in the water and evaluation of the effect of the protective coating. The behaviours of the sensors with the sensing resistors buried in the LTCC diaphragm are presented and the experiments with the drops of water, which is among the most critical situations are discussed. Performed exper- 


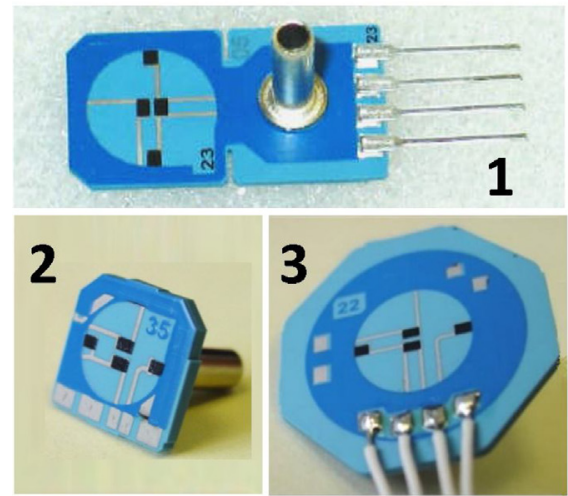

(a)
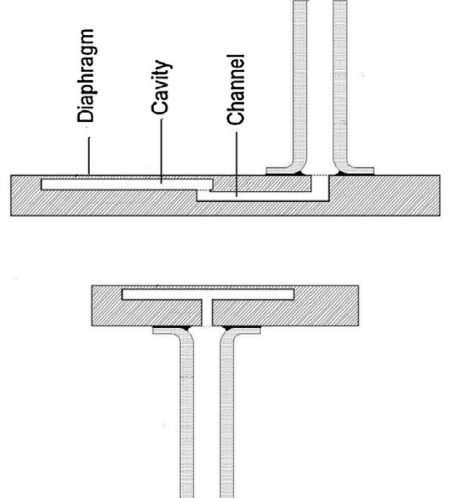

(b)

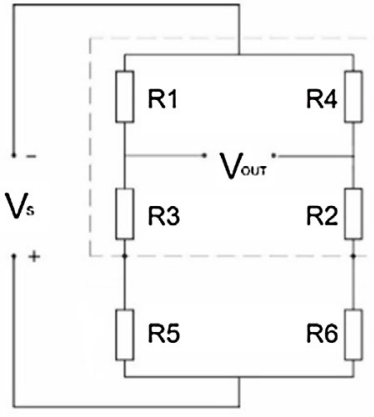

(c)

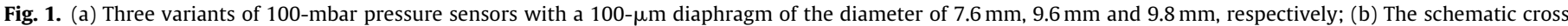
sections, not to scale (c) The Wheatstone-bridge connection of the sensing resistors $R_{1}-R_{4}$ and the resistors $R_{5}$ and $R_{6}$ used for balancing the bridge.

iments can be useful in forecasting sensor operation in condensing environments and providing appropriate activities in the context of proactive system maintenance.

\section{Considered LTCC sensors and test structures}

For this study we selected three slightly different versions of a thick-film ceramic pressure sensor in Wheatstone bridge configuration designed for relative pressure measurements in the range up to $100 \mathrm{mbar}$ (Fig. 1). These are variants of the basic sensor structure, which may be supplemented by the corresponding electronics for sensor signal processing and calibration (directly on the LTCC substrate or by an external circuit). The sensors have a $100 \mu \mathrm{m}$ thick diaphragm and almost the same layout of the sensing resistors $R_{1}$ $\mathrm{R}_{4}$. The differences are mainly in the dimensions and the outer form of the ceramic structure, in the layout of the resistors $R_{5}$ and $R_{6}$ which were specially designed for balancing the bridge and the layout of the conductor lines and electrical connections. The difference is also in the realization of the pressure channels and the pressure inlets which in general may affect the sensor's characteristics but are not considered to be critical from the perspective of the environmental effects.

The sensors have been manufactured by using Du Pont 951 green tape, the conductor paste DuPont 7484 (Pd/Ag) and the thick-film resistor materials Du Pont 2041 and 2031 for the bridge resistors $R_{1}-R_{4}$ and $R_{5}-R_{6}$, respectively. They were partially or completely covered with different protective coatings for hybrid thick-film circuits. The offset voltage $\left(\mathrm{V}_{\text {off }}\right)$ is balanced by active laser-trimming of resistors $R_{5}$ and/or $R_{6}$ to the value $V_{\text {out }}< \pm 200 \mu \mathrm{V}$ and after $24 \mathrm{~h}$ stabilisation the offset typically remains within the limits of $\pm 100 \mu \mathrm{V}$, which is acceptable in practice. After trimming, the resistors used for balancing the bridge and the electric connection lines were covered with ESL 240-SB, a screen printable thermosetting modified silicone protective coating. For this reason these parts are not visible in Fig. 1(a).

For carrying out experiments with the sensors operating in the water, the test sensors of variant 1 with irrelevant minor changes in the design were fully covered with the protective coating. For comparison, the sensors with the sensing resistors buried under an additional $50-\mu \mathrm{m}$ LTCC film were also manufactured. Both series were made by using Du Pont 2041 material for all resistors $\left(R_{1}-R_{6}\right)$ and active trimming was not performed. The samples of both sensor series are presented in Fig. 2. Notice, that the overall thickness of the diaphragm in the case of protection with the additional LTCC layer is $150 \mu \mathrm{m}$ and the sensing resistors are not on the surface of the

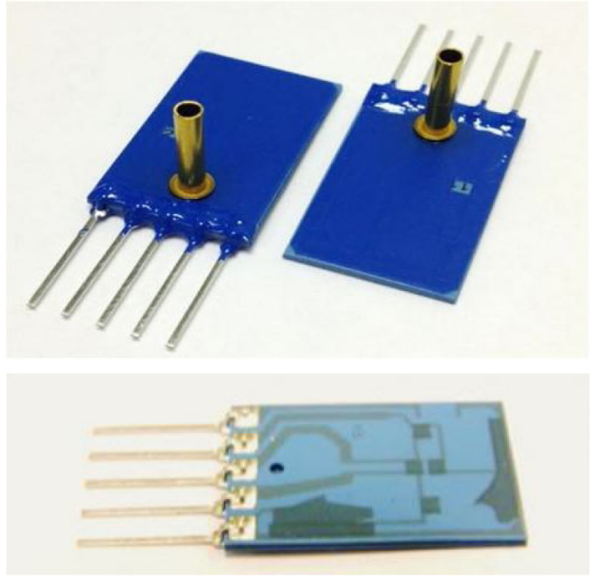

Fig. 2. The sensors coated with the protective coating (up) and the sensor with the sensing resistors buried in the LTCC diaphragm (bottom).

diaphragm (position of the maximum strain) so that the sensitivity of those sensors is accordingly lower.

For experimentation with droplets of water we used a simple test structure with the same dimensions and the layout of the sensing elements as in the case of the sensor variant 3 but with an open access to the sensing diaphragm on both sides (Fig. 3).

The sensors 1-3 were initially characterised for operation in the air in the temperature range from $-25^{\circ} \mathrm{C}$ to $75^{\circ} \mathrm{C}$ at the relative humidity of $40 \%$. Typical sensitivity (S) is in the range of $13.5 \mu \mathrm{V} / \mathrm{V} / \mathrm{mbar}$ to $14.5 \mu \mathrm{V} / \mathrm{V} / \mathrm{mbar}$, depending on the diameter of the diaphragm. The linear coefficient of the temperature dependence of sensitivity is about $500 \times 10^{-6} /{ }^{\circ} \mathrm{C}$. The $\mathrm{V}_{\text {off }}$ is within a few tenths of micro Volts (up to $\pm 100 \mu \mathrm{V}$ ) and its temperature dependence is typically less than $20 \mu \mathrm{V} /{ }^{\circ} \mathrm{C}$. As an example, the measured characteristics of the sensors of variant 3 are shown in Fig. 4. The long term stability of the $\mathrm{V}_{\text {off }}$ is typically less than $2 \%$ of the full scale output (FSO).

\section{Application of different environmental conditions}

\subsection{Impact of specific environmental conditions}

From the perspective of long-term stability we should be aware of drift in resistance value when the thick-film resistors are subjected to changes in temperature and/or humidity. In the Wheatstone bridge connection of four equal resistors the aging effects of thick-film resistors as well as other changes in the 


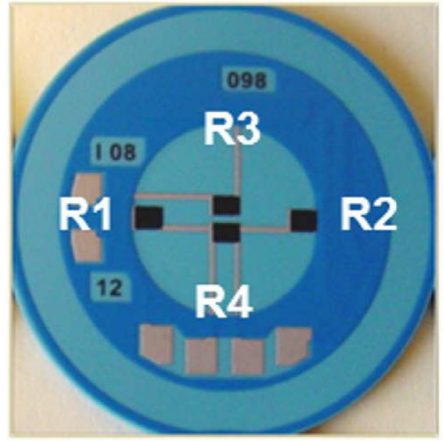

(a)

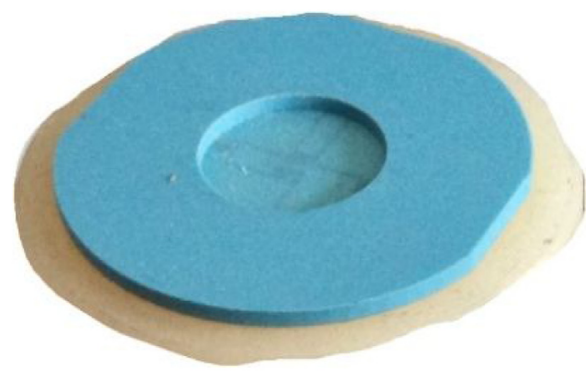

(b)

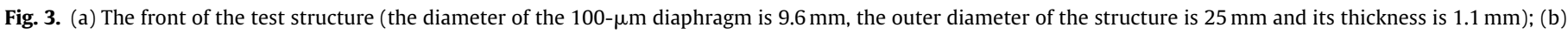
The back side of the test structure.

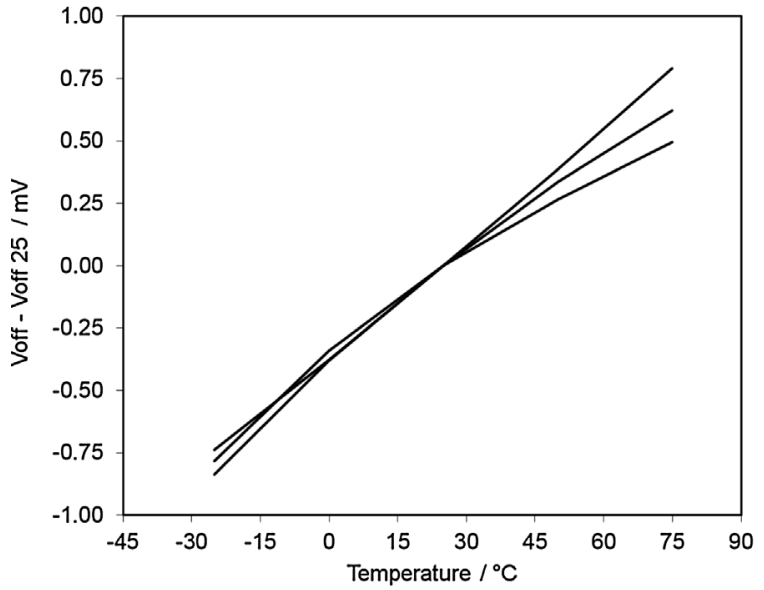

(a)

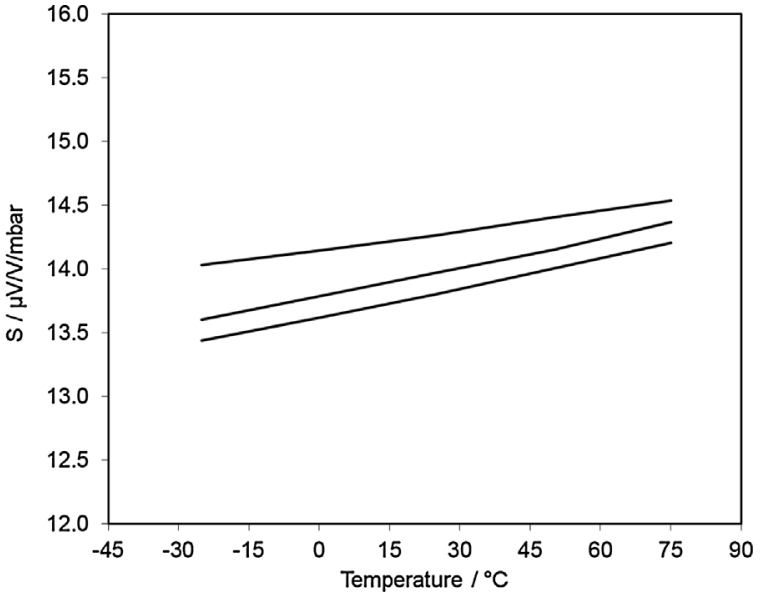

(b)

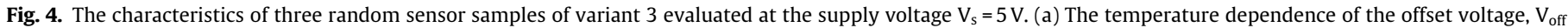
$-\mathrm{V}_{\text {off25 }}$, where $\mathrm{V}_{\text {off25 }}$ denotes the offset voltage measured at $25^{\circ} \mathrm{C}$; (b) The temperature dependence of sensitivity.

resistivity might be in an ideal case cancelled out. But since the technology restricts the accuracy of thick-film resistors to the order of $10 \%$ of the nominal value, the sensors have normally nonzero offset. Moreover, the resistors are located at different positions on the diaphragm wherein the self-heating effect is different and for this reason their resistances differ slightly, which is also reflected in the offset voltage. Balancing the bridge by trimming additional resistors can set the offset within the required range at the room ambient conditions but may not sufficiently compensate the changes due to the aging and/or environmental influences. After trimming, the resistors may be less stable, especially if they are of small dimensions and are exposed to a harsh environment (higher temperature and/or humidity), [13]. In this context, it can be expected that the influence of harsh environmental conditions may be more pronounced.

Depending on the substrate material, resistor size, type of conductor terminations, type and extent of the laser cut, the high performance thick-film resistors exposed to the accelerated aging conditions (in accordance with the common criteria for long-term storage) typically exhibit maximum resistance changes of less than $0.75 \%$ and often less than $0.2 \%$ [14]. However, in the cases, where the bridge resistors do not change proportionally, the offset voltage will change non-negligibly even in the case of an order of magnitude smaller relative changes in the resistances. (For example, in the case of sensor 1 from Fig. 1, the change of one resistor for trimming the bridge by $0.2 \%$ causes a change in the offset voltage of $6 \%$ FSO, which is often not negligible in practice.)
The impact of the environment on the offset voltage also depends on the layout of the conductor lines and the resistor terminations, particularly when the lines between the bridge resistors are longer and contribute non negligible resistance. Beside component tolerances, there is also an effect of self-heating of resistors in different locations. Consequently, it is difficult to precisely foresee how the sudden changes in the external conditions (such as the immersion of sensor in the water) may change the offset. Moreover, the protective coating may also influence the cooling conditions of the self-heated resistors and thus affect the offset response. Longlasting exposure of some protective coatings to humidity (due to the coating permeability and absorption of humidity and swelling) may result in the change of offset.

In the following we refer to various experiments performed in the design phase of the LTCC pressure sensors. In these experiments the sensors were exposed to high humidity and were even immersed in the water. We focused primarily on observation of the effects on the offset voltage, because the stability of this parameter is often critical for the applications in practice.

\subsection{Experimental work}

\subsubsection{Operation in a humid environment and in the water}

In order to inspect closely the behaviour of the sensors in the humid atmosphere and evaluate the stability of the offset voltage in terms of relative humidity $(\mathrm{RH})$ the sensors were first characterised under controlled environmental conditions in the 


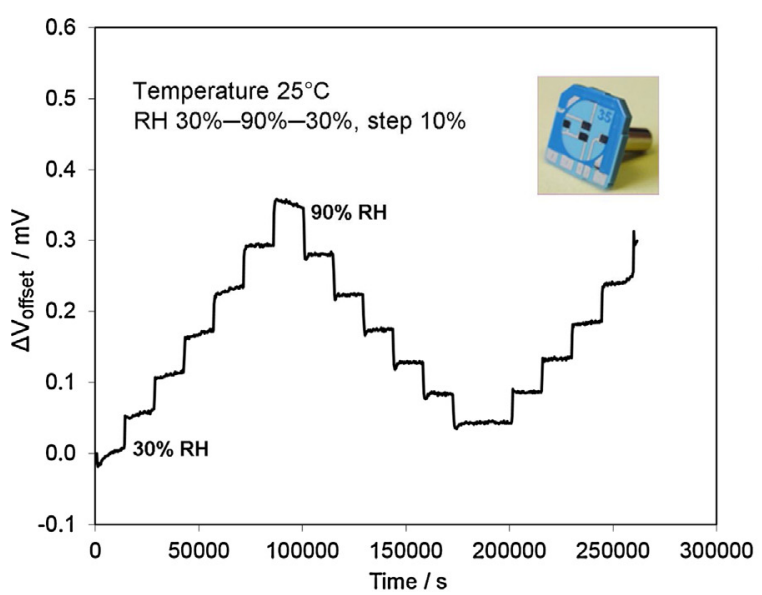

(a)

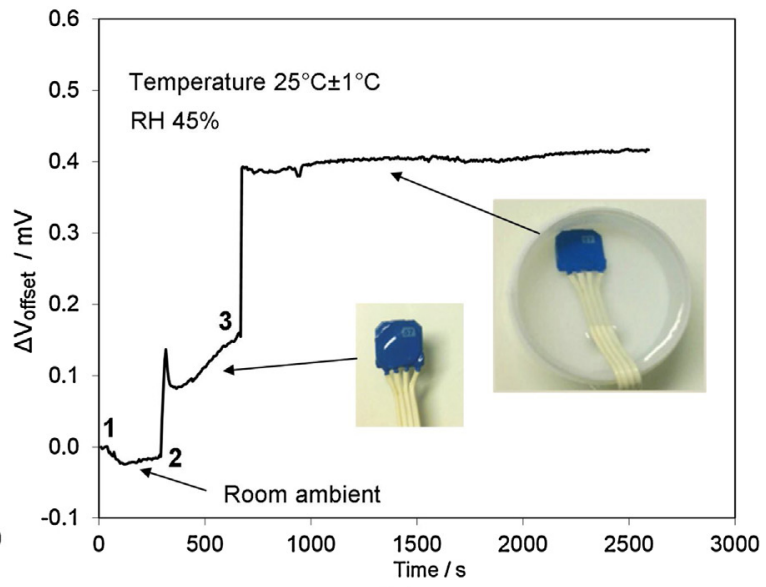

(b)

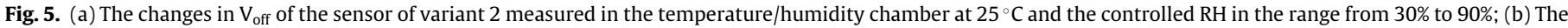
changes in $V_{\text {off }}$ resulting from the application of the water drop on the sensor surface and after immersing the sensor into the water.

temperature/humidity chamber. The measurements were carried out at the constant temperature of $25^{\circ} \mathrm{C}$ and the time controlled RH-cycles in the range of 30-90\% RH with a step of $10 \%$ RH (with a 2-h stabilisation at each step). The results obtained for the random sensor of variant 2 are presented in Fig. 5(a). The graphs below show the changes in the offset voltage $\left(\Delta \mathrm{V}_{\text {out }}\right)$ measured at least 30 min after connecting to the power supply, i.e. when the sensors are already warmed up and stabilized. As evident from Fig. 5(a), during the initial RH cycle $\mathrm{V}_{\text {off }}$ changed for up to $350 \mu \mathrm{V}$ (i.e., about $5 \mu \mathrm{v}$ per\% $\mathrm{RH}$ ). There has also been a small hysteresis, which for operation at normal ambient conditions ( $\mathrm{RH}$ of $40 \%$ up to $70 \%$ ) in principle is not critical since it is within the acceptable limits for the long term stability of the offset. However, we should be aware of this effect and take it into account if the sensor operates in an environment that is dynamically changing.

For further observations, the samples of the same sensor variant were covered with a protective coating allowing us to immerse them in the water. The offset voltage was first measured in the air at the normal ambient conditions (temperature of $25^{\circ} \mathrm{C}$ and $40 \% \mathrm{RH}$ ). Without interrupting the measurements we then applied a drop of the water on the diaphragm and after some time the entire sensor was completely immersed in the water. The results are presented in Fig. 5(b).

In the next step we explored how a direct contact with the water affects the characteristics of the sensors protected with the protective coating and the sensors with the sensing resistors buried under $50-\mu \mathrm{m}$ LTCC layer. Due to the higher thickness of the diaphragm and the reduced strain at the location of the resistors under the surface of the diaphragm, the sensitivity of sensors with the buried resistors is accordingly lower. However, these sensors also respond to the changes in the conditions when immersed in the water. For this case study we used the sensors shown in Fig. 2. The sensors were plunged into the water up to the level where the whole diaphragm is in the water with the unprotected electrical contacts above the water surface. The situation is schematically presented in Fig. 6(a).

In order to measure several sensors simultaneously we have built an experimental setup shown in Fig. 6(b) with the sensors protected by the coating (in front) and the sensors with the sensing resistors buried in LTCC (behind). After immersion in the water the offset voltage normally changes to some extent and then stabilizes at a slightly modified value in a few minutes. The results obtained by continuous measurements of three random samples, which were first stabilized in the air and then immersed in the water at the room ambient conditions are presented in Fig. 7.
It is evident that independently of whether the sensor is protected by a coating or with an additional LTCC layer, the contact with the water always has a non-negligible impact on its response. The offset voltage immediately changes to some extent even if only a drop of the water is dispensed on the diaphragm or the sensor is completely immersed in the water. The above measurements showed the change in $\mathrm{V}_{\text {off }}$ of a few tenths to several hundred $\mu \mathrm{V}$, which represents several\% of FSO. (The change in $V_{\text {out }}$ of $500 \mu \mathrm{V}$ for the sensor of variant 2 represents about $7 \%$ FSO, and is equivalent to the response to the compressive loading of about 7 mbar or the changes in the temperature of the surroundings as much as $25^{\circ} \mathrm{C}$.)

Moreover, the sensors with different protective coatings have different behaviours. The coating may decrease the sensor sensitivity (depending on the material elastic properties) and the offset is also affected because the coating has different thermomechanical properties and can absorb moisture after a prolonged exposure to a humid atmosphere/media. For operation with the known measured media at the anticipated, constant or slowly changed environmental conditions (for example all the time in the water) this effect should not be problematic and can be taken into account when designing/selecting the sensor for operation in such conditions. However, one should be aware that when the humidity is dynamically changed and the surrounding and/or measured media is a mixture of air and moisture drops the sensor may not to operate according to specifications.

The preliminary observations [10] revealed a slightly different response of the sensors covered with another Room-TemperatureVulcanizing (RTV) protective coating. Different coatings have different moisture absorption capabilities and may have very different moisture penetration properties so that the observed effect can come from the parasitic resistances originating from the protective coating. An ideal protective coating for pressure sensors is flexible (possess a low modulus of elasticity) and thin enough not to reduce the pressure sensitivity. For this reason, the siliconebased coatings with the lower modulus of elasticity may be more appropriate than the epoxies or other harder RTV conformal coatings. However, the problem is that although silicone is one of the most hydrophobic molecular species the silicone coatings allow fast moisture penetration and are not necessarily the best protection for operation in the water $[15,16]$. Silicone coating allows almost 10 times or more moisture to penetrate than typical acrylic conformal coating and permits the corrosive elements such as sulphur dioxide, sodium and chloride ions to be carried along onto the surfaces under the coating. High moisture permeability is the main disadvantage of the silicone-based conformal coatings 


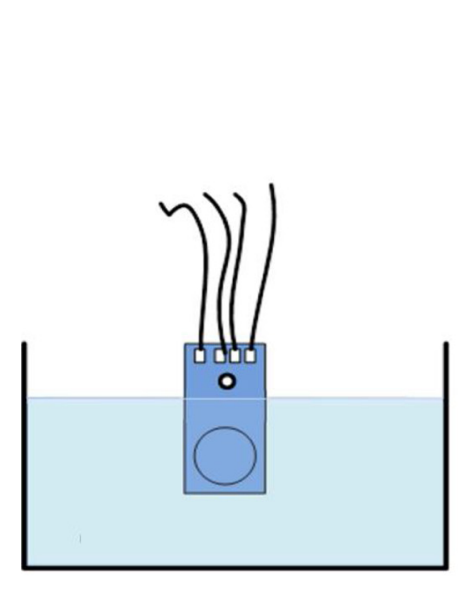

(a)

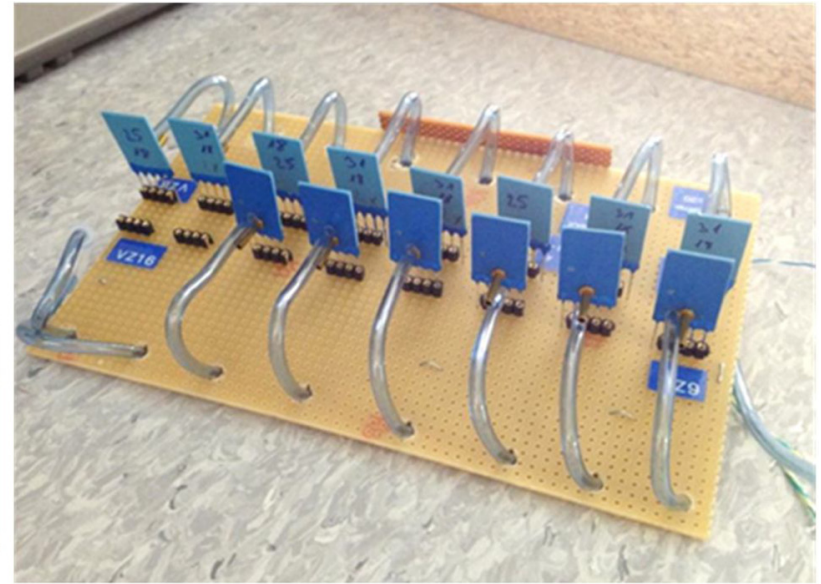

(b)

Fig. 6. (a) The schematic presentation of the sensor immersed in the water, (b) Experimental set-up for simultaneous measurements of the sensors immersed in a liquid.

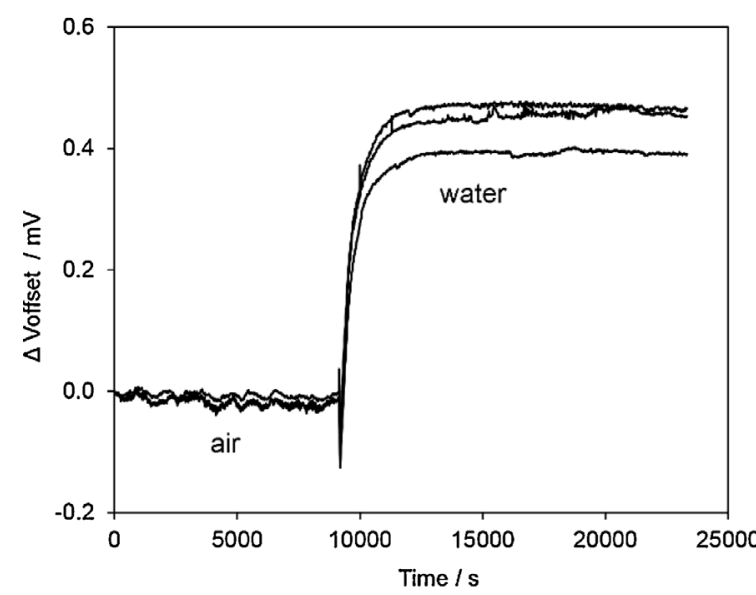

(a)

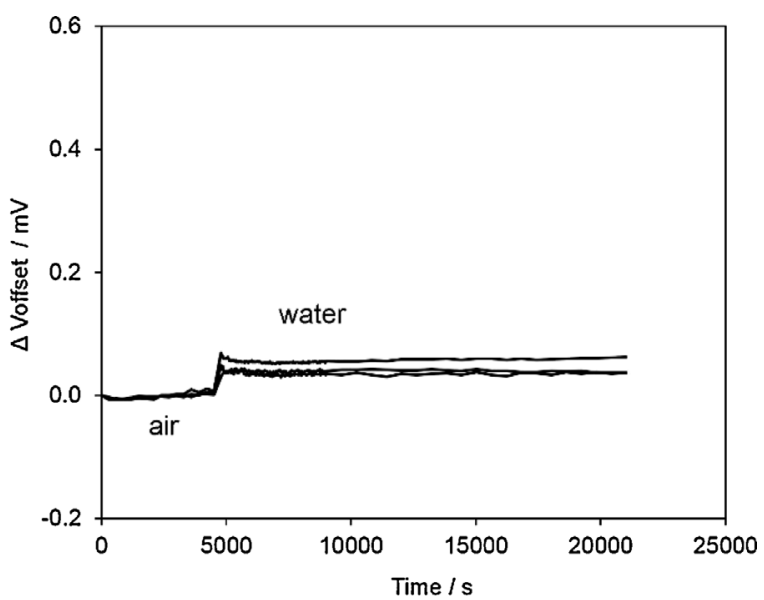

(b)

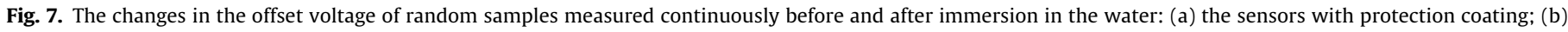
sensors with LTCC film over the sensing diaphragm.

from the perspective of protection for improvement of the sensor lifespan (durability) when they are normally operating in harsh environment (or at harsh storage conditions). In our experience, silicone-based coatings may be problematic even when used as protection against occasional/accidental short contacts with the moist environment or measured media, (e.g. air with the drops of the water or other electrically conductive fluids). The value of $\mathrm{V}_{\text {off }}$ after stabilisation depends on the changes in the bulk resistivity of the coating (due to absorption of the water), which contribute parasitic resistance to the functional resistors and the conductive lines. Our experiments showed that the extent of these parasitic effects and the dynamic changes in $\mathrm{V}_{\text {off }}$ also depends on the coating thickness. This impact could originate from the increase of the mass of the water logged coating and mechanical stress due to its increased volume on the deflection of the diaphragm. Stabilisation of the $\mathrm{V}_{\text {off }}$ measured for the sensors with the silicone-based protective coating of different thicknesses $(20 \mu \mathrm{m}$ and $40 \mu \mathrm{m})$ after immersion in the water in comparison to the $V_{\text {out }}$ of the sensor with an additional LTCC layer over the sensing diaphragm is shown in Fig. 8. The time dependence of the changes in $V_{\text {off }}$ seems to be similar to the typical water absorption curves (percentage change in mass versus time) obtained by dynamic vapour sorption (DVP) method [17]. This similarity suggests that the changes in $\mathrm{V}_{\text {out }}$ come mainly from the increase in the thickness of the coating which rep-

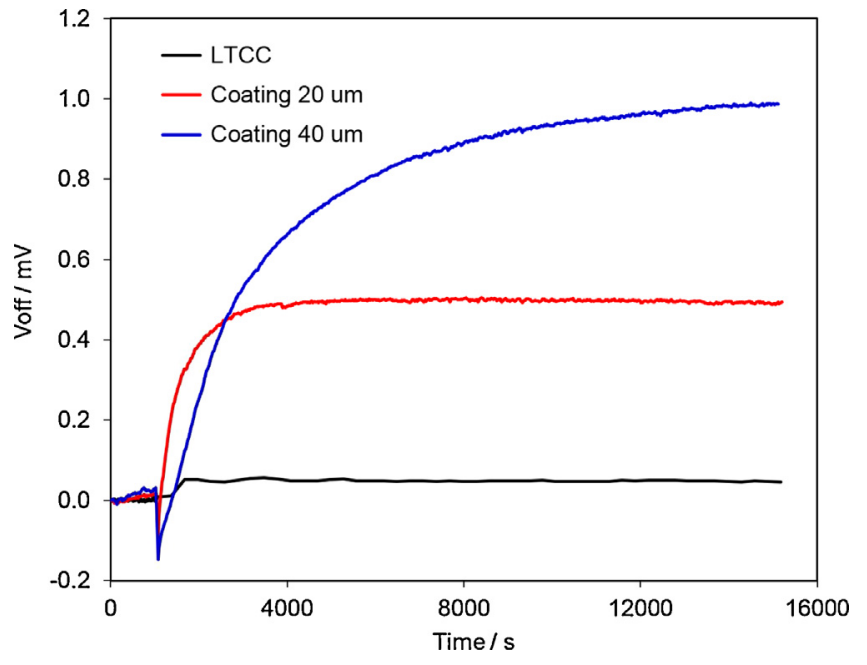

Fig. 8. Typical responses of the sensors of variant 1 with silicone-based protection of two different thicknesses and the sensor with additional LTCC layer after immersion in the water.

resents a significant part of the total thickness of the diaphragm. The increased thickness of the coating due to the absorption of water 
causes the changes in the strain components as well as the thermal state of the sensing resistors and consequently affects the sensor's characteristics.

The measurements of the sensors immersed in the water showed typical changes in the sensitivity in the range of $1-3 \%$. These changes are mainly resulting from the combined effects of non-uniform hydrostatic pressure and the changed thermal state of the sensing resistors. After subtracting the effect of hydrostatic pressure (the sensor is immersed in the water about $14 \mathrm{~mm}$ deep and the centre of the diaphragm is approximately $8 \mathrm{~mm}$ above the water level) the changes in the sensitivity remain at about $1 \%$. The response of the sensor with the $20-\mu \mathrm{m}$ coating to the air pressure loads in the range from -50 mbar to 50 mbar is presented in Fig. 9. Fig. 9(a) shows the characteristics obtained for the sensor measured at three different situations. Initially the sensor was measured during operation in the air (i.e. after stabilisation at the normal ambient conditions). Than it was immersed in the water and measured two times: immediately after immersion and once again after $15 \mathrm{~min}$. The measurements showed that the drift in the sensors' response when immersed in the water also depends on the pressure applied to the sensor and the hydrostatic pressure acting in the specific case of the experimental set-up. The situation is clearly evident from Fig. 9(b) which shows the difference between the measurements in the air and in the water.

In the next step, we explored the behaviour of the sensor in some specific situations, namely in the case when the drops or/and small amounts of the water accidentally appear on the diaphragm.

\subsubsection{Sensors' response to drops of water}

For experimentation with drops and small streams of the water we used the above described test structure shown in Fig. 3. In order to explore the sensor's response the output voltage $\left(V_{\text {out }}\right)$ was continuously measured during the experiment in which drops of the water were dispensed on the diaphragm (the opposite side of the thick-film resistors) and left there to evaporate at the room ambient conditions until the sensor surface was not completely dry. The acquisition of the measurement results started after the sensor was warmed up and completely stabilized, i.e. at least $60 \mathrm{~min}$ after its connection to the supply voltage $V_{S}=5 \mathrm{~V}$. The measurement data were recorded every $7 \mathrm{~s}$. After measuring a few minutes of stable operation without any loads, drops of the water (with the volume of about $8 \mu \mathrm{l}$ ) were one by one dropped in the middle of the diaphragm. When the mass of the cumulated drops was big enough, the drop was spilled over the whole membrane. The response to the characteristic situations shown in Fig. 10(a) is presented in Fig. 10(b).

The characteristic segments of the measured $V_{\text {out }}$ are as follows:

- Stabilized normal operation at $\mathrm{V}_{\mathrm{S}}=5 \mathrm{~V}$, (point 1 );

- One drop of the water was applied on the central part of the diaphragm (point 2) and, as a consequence, $\mathrm{V}_{\text {out }}$ immediately increased (point 3);

- After applying three drops, which correspond to three peaks (point 3)the water spilled over the entire membrane (point 4);

- Drying: the central part of the diaphragm is dry (point 5) and drying slowly continues towards the diaphragm edge;

- The narrow region at the edge of the diaphragm is covered with the evaporating water (point 6);

- Stabilized operation of the dry structure (point 7).

Since the mass of the water drops is too small to cause a change in $\mathrm{V}_{\text {out }}$ as a result of mechanical load, a possible reason for the obtained results could be the dynamic change in the temperature distribution due to the Joule heating of the thick-film resistors and the local cooling due to the evaporation of the water. The first peak in the measured characteristics results from the instantaneous local change in the temperature of the diaphragm in its central part when the drop of the water touches the diaphragm.

The experiment was repeated several times on the same and also other test samples. Each time, the response obtained for approximately the same amount of the water was similar to the characteristic shown in Fig. 10(b). The diaphragm starts to dry at the central part, where it is heated by the resistors $R_{3}$ and $R_{4}$. The water remains longest at the border of the diaphragm. Since LTCC ceramic has closed pores the moisture remains on the surface of the structure. The biggest volume of the drop before it pours over the diaphragm and how long the moisture can remain on its surface also depend on the surface roughness. Typical roughness of LTCC (according to the datasheet) is $0.35 \mu \mathrm{m}$. Our measurements of the diaphragm surface roughness showed $R_{a}<0.45 \mu \mathrm{m}$.

For another experiment, a thin film of gold (thickness of about $20 \mathrm{~nm}$ ) was sputtered onto the rear of the diaphragm. This has changed the roughness of its surface and the effective thermal conductivity. Consequently, the response of the structure to the small stream of the water slightly changed. For comparison, the responses of two sensors from the same series, one of them covered with the sputtered gold, are shown in Fig. 11.

The response of the sensor without additional Au layer (Fig. 11(b)) is similar to that in the example above (Fig. 10). The difference in the peak (point 3 ) is because this time the droplets were dispensed on the diaphragm immediately one after the other, without intermediate time intervals until the water has spilled over the entire surface of the diaphragm. The response of the sensor with the sputtered $\mathrm{Au}$ (Fig. 11(a)) differs from the response of the sensor without Au shown in Fig. 11(b) in the time interval between points 5 and 6 . One the possible reasons may be that due to the slightly higher thermal conductivity of the diaphragm coated with Au, the effect of the local heating of the central resistors is less pronounced. The time interval in which the dry area of the diaphragm extends toward the edge is slightly longer and at the end of this interval the $\mathrm{V}_{\text {off }}$ characteristic is more wedge-shaped.

\section{Results and discussion}

An extremely humid atmosphere and occasional presence of water drops is among the most problematic environment/media for pressure sensors. In general, the pressure sensors are protected against harmful effects of media either with the protective coatings or are designed in the appropriate housings to avoid direct contact of the sensing elements with the environment and/or measured media. The protection is necessary for silicon MEMS pressure sensors, while the LTCC technology enables manufacturing of sensor structures that may operate in water or different harsh media without any special protection. Only the electrical connections and the readout electronics added to the thick-film sensing elements need to be protected. However, there may also be cases where due to the technological limitations (e.g. manufacturing of the diaphragm thinner than $150 \mu \mathrm{m}$ ) it is necessary to use a protective coating.

Our experiments have shown that the protective coating itself may impact the response of the sensor when it is exposed to a humid environment or immersed in the water. Attention must be paid with silicone-based protection, which absorbs water and thereby increases the thickness. The absorption of water results in changed thermal conductivity and the electrical resistance of the coating. Increasing the thickness of the protective coating affects both the strain components and the thermal state in the sensing resistors. Such changes affect the offset voltage, which may also exhibit a small hysteresis as shown in Fig. 3(a). This is due to accumulation of the moisture absorbed by the protective coating during high $\mathrm{RH}$ cycles, which remains in the coating much longer than the duration of the experiment. The influence of the absorption of 


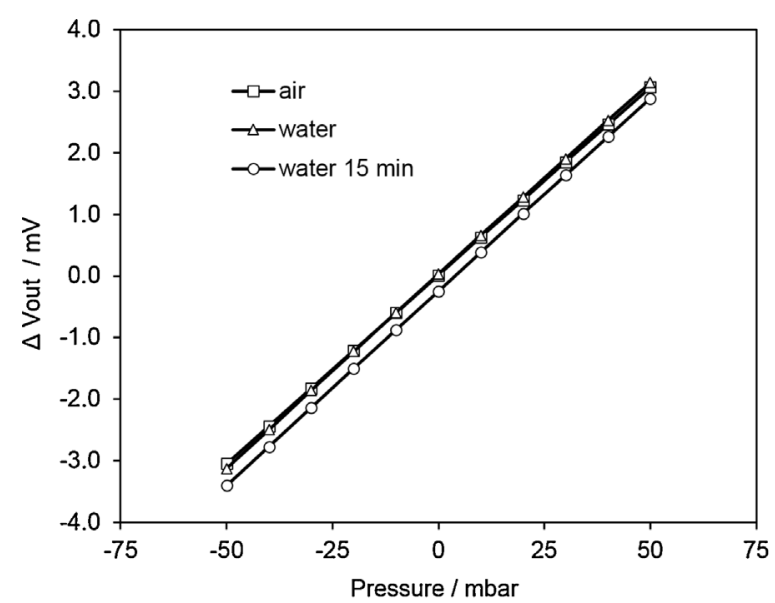

(a)

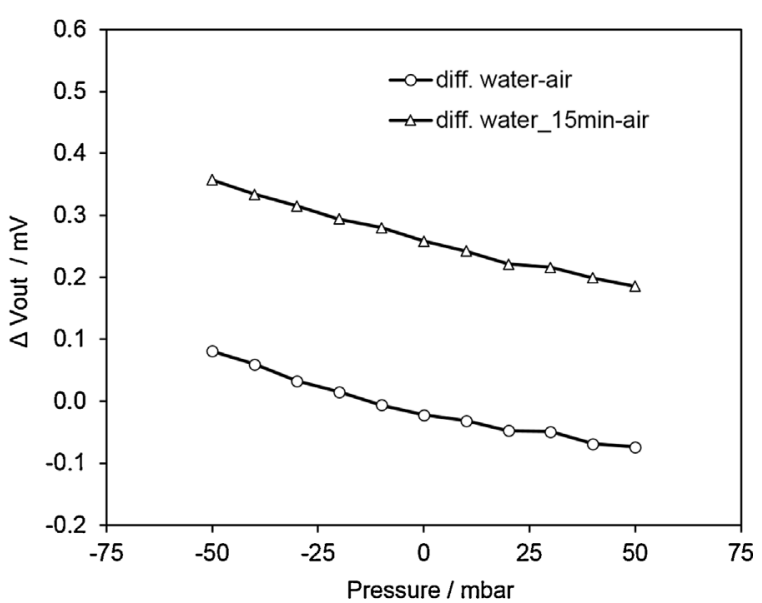

(b)

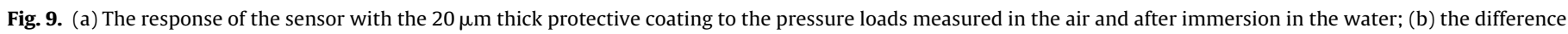
between the readings in air and in the water.

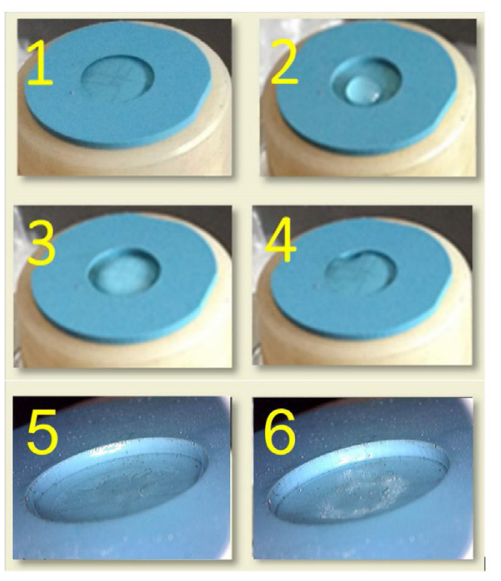

(a)

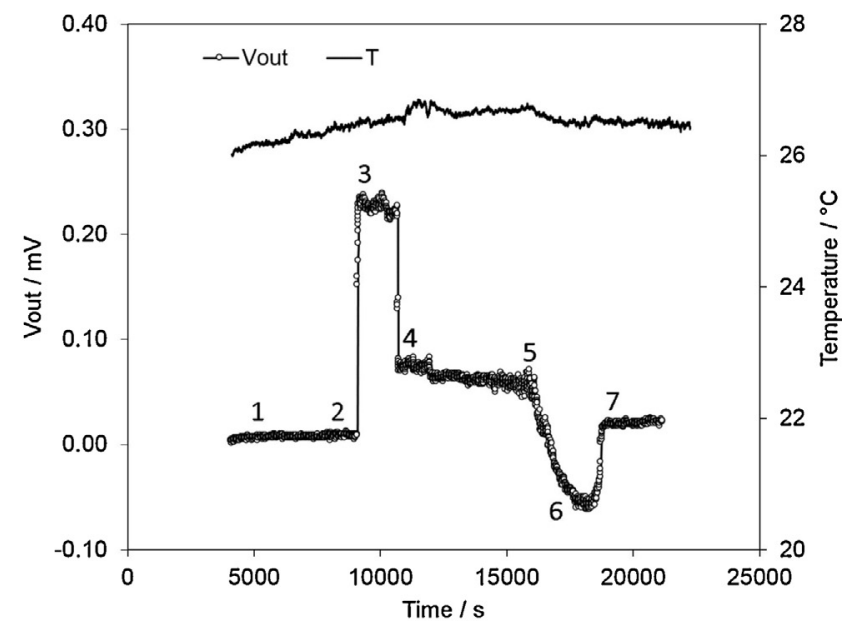

(b)

Fig. 10. (a) The specific situations of water drops on the diaphragm; (b) The measured $V_{\text {out }}$ and the ambient temperature in the vicinity of the test structure.

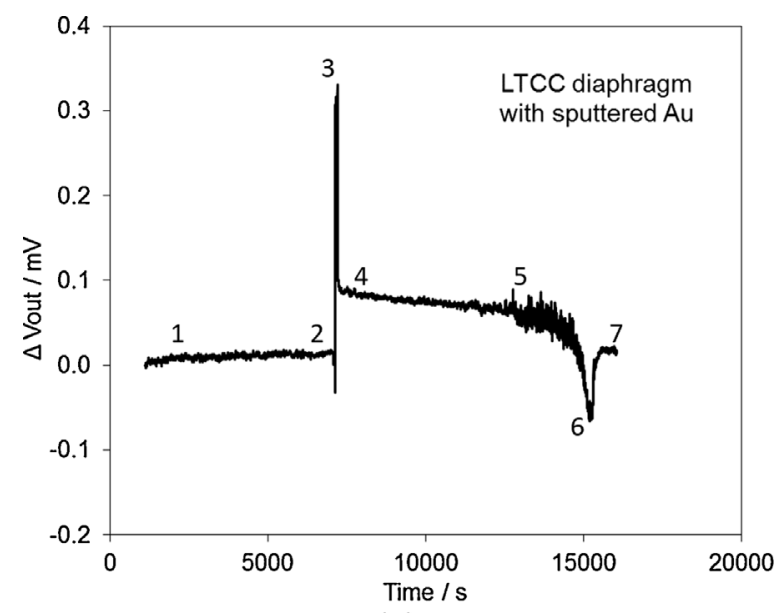

(a)

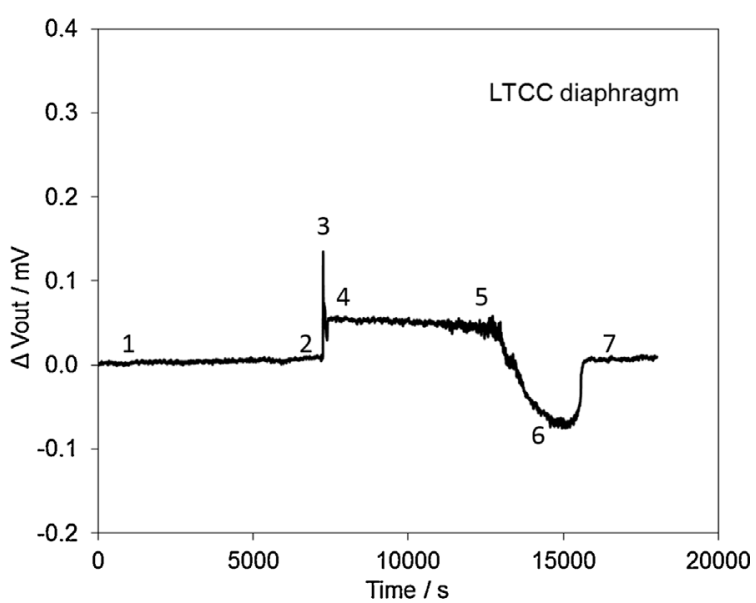

(b)

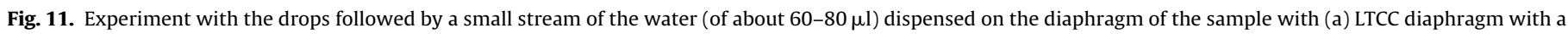
thin film of sputtered Au, (b) diaphragm without Au.

water is most evident when the sensors are completely immersed in the water. The measurements revealed the dependence of the effect of the thickness of the protective coating. This problem could be solved with the use of Parylene conformal coating [17,18], but 
this technology is more expensive and it also has some limitations when used for protection of the 3-D structures with cavities and channels. From the above it is clear that the preferred sensor embodiment is entirely made of ceramic, so that its functional part may be immersed in the medium with no protection. However, the use of protective coating in the case of very thin diaphragms may not always be a feasible solution.

The humid air with possible appearance of dew may influence the response of the sensor in the real-time operation because of the changes of the thermal conditions in the sensing structure. Due to the Joules heating the thick-film resistors with similar resistances, which are placed at the different locations on the diaphragm, have slightly different temperatures. When such sensing structure is immersed in the water or some other liquid media the temperature of the individual resistors changes depending on the temperature of the media and its temperature conductivity. The resulting resistance changes reflect in the response of the sensor. Similarly, water drops on the diaphragm cause local cooling of the sensing resistors which results in the changes of $\mathrm{V}_{\text {off }}$. As the water evaporates and the diaphragm is selectively dried, the non-uniform temperature distribution influences the offset voltage.

\section{Conclusions}

The experiments presented in this paper give insight into changes of characteristics of piezoresistive LTCC sensors in dynamically changing condensing environment. Performed measurements indicate that sensors' short-term reactions are non-negligible and should be considered throughout the entire span of the sensor life cycle. Knowledge of these mechanisms can help us not only in planning but also in the selection of appropriate sensors in target system applications and their maintenance. The latter is of particularly importance for defining strategy and goals of proactive system maintenance in harsh conditions. Understanding the origin of dynamic changes in sensor responses may improve system control algorithms. We focused our work on LTCC sensors because we have most experience in their development and design. However, we believe that similar conclusions can be reached also with other technologies.

\section{Acknowledgments}

This work was partially funded from the Electronic Component Systems for European Leadership Joint Undertaking under grant agreement No 662189 (MANTIS) [19]. This Joint Undertaking receives support from the European Union's Horizon 2020 research and innovation programme and Spain, Finland, Denmark, Belgium, Netherlands, Portugal, Italy, Austria, United Kingdom, Hungary, Slovenia, Germany. The authors also acknowledge the financial support from the Slovenian Research Agency [research core funding No. P2-0098].The authors would like to thank Mr. Darko Belavič and Mr. Mitja Jerlah (Hipot-RR c/o CoE Namaste) for designing and manufacturing the test structure used in this case study.

\section{References}

[1] Jacob Fraden, Handbook of Modern Sensors: Physics, Designs, and Applications, 3rd ed., Springer-Verlag, New York, 2004.

[2] M.R. Gongora-Rubio, P. Espinoza-Vallejos, L. Sola-Laguna, J.J. Santiago-Aviles, Overview of low temperature co-fired ceramics tape technology for meso-system technology (MsST), Sens. Actuators A: Phys. 89 (2001) 222-241.
[3] L.J. Golonka, K. Malecha, LTCC fluidic microsystems, J. Microelectron. Electron. Compon. Mater. 42 (2012) 225-233.

[4] D. Jurków, T. Maeder, A. Dąbrowski, M.S. Zarnik, D. Belavič, H. Bartsch, J. Müller, Overview on low temperature co-fired ceramic sensors, Sens. Actuators A: Phys. 233 (2015) 125-146.

[5] Y. Fournier, T. Maeder, G. Boutinard-Rouelle, A. Barras, N. Craquelin, P. Ryser, Integrated LTCC Pressure/Flow/Temperature multisensor for compressed air diagnostics, Sensors 10 (2010) 11156-11173.

[6] G.J. Radosavljević, Lj.D. Zivanov, W. Smetana, A.M. Marić, M. Unger, L.F. Nađ, A wireless embedded resonant pressure sensor fabricated in the standard LTCC technology, IEEE Sens. J. 9 (no. 12) (2009) 1956-1962.

[7] U. Partsch, C. Lenz, S. Ziesche, C. Lohrberg, H. Neubert, T. Maeder, J. Microelectron, Electron. Compon. Mater. 42 (No. 4) (2012) 260-271.

[8] W. Missal, J. Kita, W. Eberhard, F. Gora, A. Kipka, T. Bartnitzek, F. Bechtold, D. Schabbel, B. Pawlowski, R. Moos, Miniaturized ceramic differential scanning calorimeter with integrated oven and crucible in LTCC technology, Sens. Actuators A: Phys. 172 (2011) 21-26.

[9] M.S. Zarnik, D. Belavic, The effect of humidity on the stability of LTCC pressure sensors, Metrol. Meas. Syst. Vol. XIX (No. 1)(2012) 133-140.

[10] M.S. Zarnik, D. Belavic, Study of LTCC-based pressure sensors in water, Sens. Actuators A: Phys. 220 (2014) 45-52.

[11] M.S. Zarnik, D. Belavic, F. Novak, The impact of housing on the characteristics of ceramic pressure sensors - An issue of design for manufacturability, Sensors 15 (2015) 31453-31463.

[12] M.S. Zarnik, M. Pavlović, D. Belavic, V. Sedlakova, J. Sikula, J. Majzner, Effect of media on the warm-up and the offset characteristic of piezoresistive ceramic pressure sensors, Proc. EDS'13, Electronic Devices and Systems IMAPS CS International Conference 2013 (2013) XXI-XXVI (Brno, Czech Republic, HEGR, O. Ed., Publisher: Vysoké učení Technické v Brně).

[13] A. Dziedzic, A. Kolek, W. Ehrhardt, H. Thust, Advanced electrical and stability characterization of untrimmed and variously trimmed thick-film and LTCC resistors, Microelectron. Reliab. 46 (2006) 352-359.

[14] R.E. Cote, in: D.D.L. Chung (Ed.), Thick Film Technology, in Materials for Electronic Packaging, Heinemann, Butterworth, 1995, Chapter 9.

[15] J.A. Carpenter, in: D.D.L. Chung (Ed.), Measurements of Properties of Materials in Electronic Packaging, in Materials for Electronic Packaging, Heinemann, Butterworth, 1995, Chapter 16.

[16] A.I. Technology, Inc., Frequently asked questions on conformal coatings http://www.aitechnology.com/products/conformal-coatings/conformalcoatings-faq, (Accessed 20 February 2017).

[17] J.J. Licari, Coating materials for electronic applications, in: Polymers, Processes, Reliability, Testing, Noyes Publications/William Andrew Inc., California, 2003, AvanTeco Whittier.

[18] A. Hardy, Protecting Sensors in Harsh Environments with Parylene, 2015, http://www.maintworld.com/Applications/Protecting-Sensors-in-HarshEnvironments-with-Parylene (Accessed 20 February 2017)

[19] Mantis project. http://www.mantis-project.eu/ (Accessed 23 March 2017).

\section{Biographies}

Marina Santo Zarnik received an M.Sc. degree in Computer Science and a Ph.D. in Electrical Engineering from the University of Ljubljana, Slovenia, in 1993 and 1998 respectively. Her research field covers different areas of ceramic microsystems and sensors. Her main research interests comprise characterization and finite-element modelling of thick-film pressure-sensor structures and ceramic electromechanical systems.

Franc Novak gained the BSc, MSc, and PhD degrees in electrical engineering from the University in Ljubljana in 1975, 1977, and 1988, respectively. Since 1975 he has been with the Jozef Stefan Institute, where he was head of Computer Systems Department (1994-2014). Since 2010 he is also full prof. at Faculty of Electrical Engineering and Computer Science, University of Maribor. His research interests are in the areas of electronic design and test, and fault tolerant computing. 